\title{
The impact of malaria parasitism: from corpuscles to communities
}

\author{
Thomas E. Wellems, Karen Hayton, and Rick M. Fairhurst
}

Laboratory of Malaria and Vector Research, National Institute of Allergy and Infectious Diseases, NIH, Bethesda, Maryland, USA.

\begin{abstract}
Malaria continues to exert a tremendous health burden on human populations, reflecting astonishingly successful adaptations of the causative Plasmodium parasites. We discuss here how this burden has driven the natural selection of numerous polymorphisms in the genes encoding hemoglobin and other erythrocyte proteins and some effectors of immunity. Plasmodium falciparum, the most deadly parasite species in humans, displays a vigorous system of antigen variation to counter host defenses and families of functionally redundant ligands to invade human cells. Advances in genetics and genomics are providing fresh insights into the nature of these evolutionary adaptations, processes of parasite transmission and infection, and the difficult challenges of malaria control.
\end{abstract}

\section{Evolutionary origin and host preferences of malaria- causing parasites}

Most cases of malaria in the world are caused by one of the four species of Plasmodium parasites that predominantly infect humans: Plasmodium falciparum, Plasmodium vivax, Plasmodium ovale, and Plasmodium malariae. Despite efforts over the past century, the global health burden from Plasmodium infections remains approximately 250-600 million episodes of malaria each year in Africa, Asia, Oceania, and Latin America $(1,2)$. Of these episodes, approximately $40 \%$ are caused by $P$. falciparum, as are nearly all fatalities attributed to malaria. Another approximately $40 \%$ of the episodes of malaria may be caused by $P$. vivax. Although such episodes do not often kill outright, they nevertheless are tremendously debilitating and are responsible for diminished learning, productivity, and economic development. Until recently, Plasmodium species native to other primates were thought to only occasionally infect humans. But this picture has now changed with the emergence of Plasmodium knowlesi, a natural parasite of macaque monkeys, in human populations of Southeast Asia. Reports from Malaysia, the Philippines, Thailand, and Myanmar suggest that $P$. knowlesi should be considered a fifth species responsible for a substantial prevalence of malaria in humans (3).

Parasites that cause malaria all develop through the same general life cycle, which alternates between the human host and anopheline mosquito (Figure 1). The cycle begins when a Plasmodium-carrying female anopheline mosquito takes a blood meal. Sporozoites inoculated into the dermis migrate to the liver where they infect hepatocytes. Over 5-16 days, depending on the Plasmodium species, each parasite grows and divides into tens of thousands of merozoite forms that then break out of individual hepatocytes into the blood, where they infect erythrocytes; alternatively, some P. vivax and P. ovale parasites can remain latent in the liver as hypnozoite forms until they activate and cause relapses of malaria months or years later. Erythrocyte-stage Plasmodium parasites undergo repetitive rounds of invasion, growth, and division in one-day (P. knowlesi), two-day (P. falciparum, P. vivax, and P. ovale), or three-day (P. malariae) periods. Parasite density expands exponentially as a consequence, leading to the symptoms and complications of malaria. P. falciparum is particularly dangerous in this phase of the life cycle, as host erythrocytes infected with its mature parasite forms avoid the spleen by seques-

Conflict of interest: The authors have declared that no conflict of interest exists. Citation for this article: J. Clin. Invest. 119:2496-2505 (2009). doi:10.1172/JCI38307. tering in capillaries and microvenules of the brain and other vital organs, a process that is not common with erythrocytes infected by other human malaria parasite species. Bloodstream parasitization can last for months if not treated, during which time some parasites continually leave the asexual replication phase and differentiate into male and female gametocytes. After these sexual stages are taken up by another anopheline mosquito ingesting a blood meal, they mate and infect the mosquito to generate thousands of progeny sporozoite forms that travel to the mosquito salivary glands, where the sporozoites wait, ready to initiate another life cycle.

What are the evolutionary origins of these sinister and highly adaptive parasites, and how did they come to be so successful in their transmission among human populations? Plasmodium parasites are members of the large Apicomplexa taxon (Figure 2), characterized by an apical complex of rhoptries, micronemes, dense granules, and other organelles specialized for host cell invasion. In addition to malaria, members of this taxon cause cryptosporidiosis, toxoplasmosis, babesiosis, cyclosporiasis, coccidiosis, and theileriosis (Figure 2). Evolutionary relationships among apicomplexan species are evident in the residual genes they carry from a plant plastid genome that was once present in a red algal cell engulfed by an ancient ancestor. The discovery of an algal symbiont of corals that carries a plastid and is closely related to apicomplexans suggested recently that apicomplexan parasitism was probably present in the earliest animals (4, 5). Modern Plasmodium species thus may have descended from apicomplexans that interacted with increasingly sophisticated immune systems over hundreds of millions of years (5). Through an evolutionary arms race between parasites and hosts, Plasmodium species have come to possess potent defenses to effectively deal with diverse mechanisms of innate and acquired immunity and therefore persist with stubborn success in their animal hosts.

Most of the approximately 200 known Plasmodium species infect particular lineages of vertebrates - primates, rodents, birds, and reptiles (6); they either never evolved in or disappeared from other major vertebrate lines. Further, Plasmodium species exhibit strong specificity for certain host species (6), with only occasional jumps between host species. One such jump is thought to account for the origin of $P$. vivax, which a recent phylogenetic analysis suggests occurred 53,000-265,000 years ago when an ancestral parasite switched host from macaque monkeys to humans (7). The increasing prevalence of $P$. knowlesi in human populations today may prove to be another example (3). 


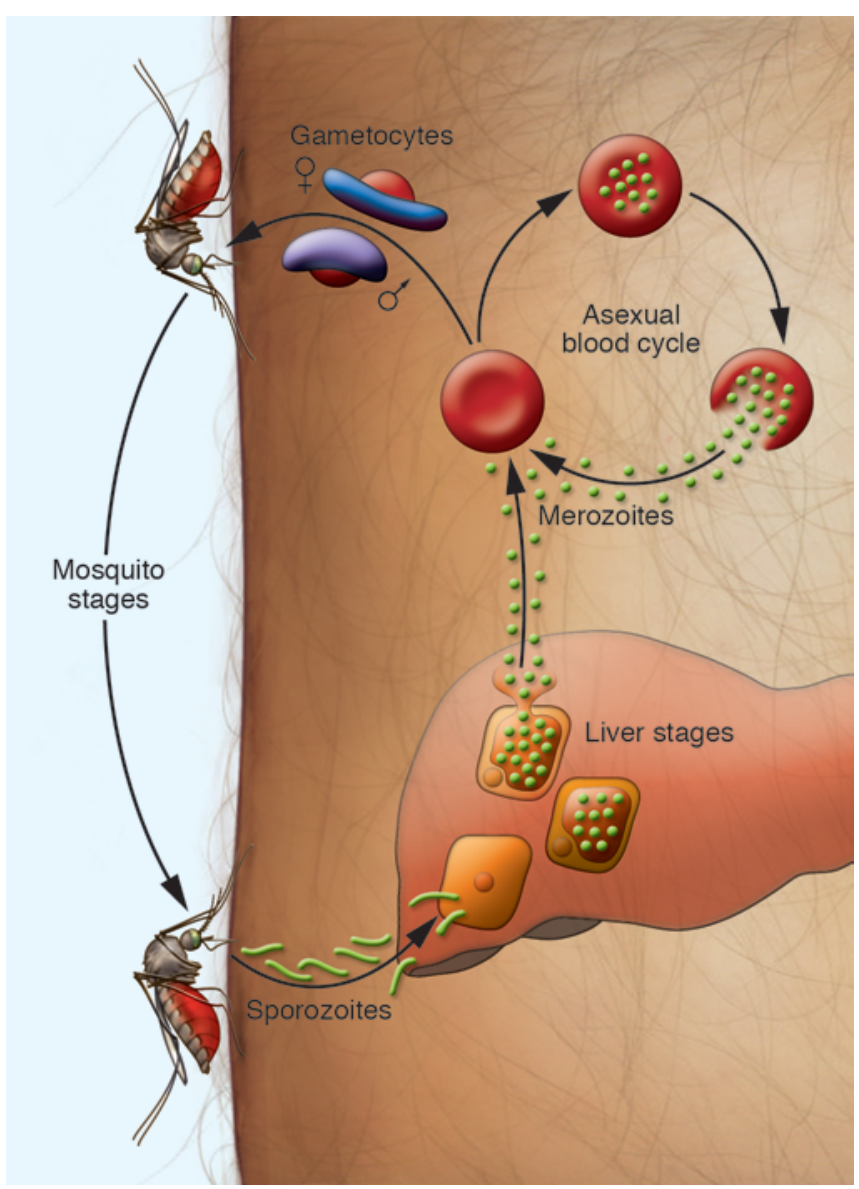

Figure 1

Life cycle of $P$. falciparum. Sporozoites injected by anopheline mosquitoes travel through the dermis and enter the bloodstream to invade hepatocytes. Each infected hepatocyte generates tens of thousands of merozoites, which then break out and reenter the bloodstream to invade erythrocytes. Numerous rounds of asexual reproduction follow, with repeated invasion of erythrocytes every 48 hours. Some parasites in the erythrocytes develop into sexual stage gametocytes, which circulate in the bloodstream and are taken up by female mosquitoes during a blood meal. In the mosquito midgut, gametes emerge from the gametocytes and cross-fertilize (118). The resulting zygote develops into an ookinete that crosses the midgut wall and grows into an oocyst. Mitotic division within the oocyst produces thousands of sporozoites that break out and travel through the hemolymph to the mosquito salivary glands, from which they are injected into a human host.

Recent advances in genetics and genomics make it possible to address key areas of malaria research with new power and detail. Among these are the host-pathogen relationships that support the extremely high burden of $P$. falciparum in Africa and reasons why most parasitized individuals avoid the complications of severe malaria while others succumb. The wealth of data on Plasmodium will help us understand the families of antigenically variant but functionally redundant ligands that malaria parasites employ to interact with host cells. As questions in these areas have been difficult to address with previously available experimental tools, approaches that employ information from the rapidly expanding databases will provide invaluable resources for state-of-the-art investigations (8-10). These can be expected to advance Plasmodium biology and support new approaches against malaria.

\section{Intense transmission of $P$. falciparum by African mosquitoes}

As might be expected of parasites that coevolved with their hosts over millions of years, Plasmodium infections in malaria-endemic regions often go unnoticed. Even in Africa, where P. falciparum accounts for nearly all deaths from malaria, most bites from infected mosquitoes do not produce clinically apparent disease. Children exposed to such bites typically have outcomes of no infection (about one-half of inoculations), infection with no symptoms (about one-quarter of inoculations), or infection with fever and other symptoms (uncomplicated malaria; about another onequarter of inoculations). Only in relatively few cases do children develop manifestations of severe malaria, which can include coma, profound anemia, and acidosis-driven respiratory distress (Figure 3A) (11). Many factors determine outcome, including mosquito inoculation rate, sporozoite dose, immunity acquired from previous exposure to $P$. falciparum, parasite virulence, host genetic polymorphisms, nutrition and housing conditions, awareness of the symptoms of malaria, and prompt access to effective treatment.

The prevalence of $P$. falciparum infection is, nevertheless, high enough to kill a million people in Africa each year, where annual death rates among young children are as high as $1 \%-2 \%$ in villages $(1,2,12)$. Death rates from malaria were even higher before the availability of modern antimalarial drugs - in past centuries, more than $20 \%$ of children may have perished in their first decade of life from malaria in areas of intense P. falciparum transmission (13). In these areas, children can be exposed to 700 or more sporozoiteinoculating mosquito bites every year (14) (Figure 3B).

Of the approximately 400 species of Anopheles mosquitoes (up to 60 of which can transmit Plasmodium species; ref. 15), African mosquitoes, particularly Anopheles gambiae, stand out because of their great anthropophily and sheer efficiency of parasite transmission. An example of the alarming potential for African mosquitoes to cause explosive epidemics occurred in the 1930s when they were inadvertently introduced from Africa into Brazil by ship or, less likely, by plane (16). The mosquitoes were able to tentatively colonize riverine and coastline regions north and west of Natal and in 1938 produced the greatest malaria epidemic ever in South America, with over 100,000 cases and 14,000 deaths. The new anopheline infestation was eliminated only after intensive efforts of the Brazilian Malaria Service and the American epidemiologists Fred L. Soper and D. Bruce Wilson. Satisfyingly, reintroduction of these mosquitoes has been successfully averted to this day (16).

In contrast with the experience in Brazil, control of An. gambiae and related mosquitoes has proven vastly more difficult in Africa, where the insect populations are highly adapted to their native habitats and have behavioral patterns highly conducive to parasite transmission. This was dramatically demonstrated in the Garki Project of northern Nigeria, 1969-1976. Vectorial capacity, a mathematical expression of the daily rate of indoor and outdoor mosquito contact, was found to be 1000 times the level necessary for maintenance of transmission, putting malaria in the African savanna in a category of its own (17). The Garki Project's intensive control operations, including mass drug administration and comprehensive spraying of domiciles with residual insecticide, were able to reduce vectorial capacity by only $90 \%$ (in the absence of insecticide-resistant mosquitoes and drug-resistant parasites) and thus 


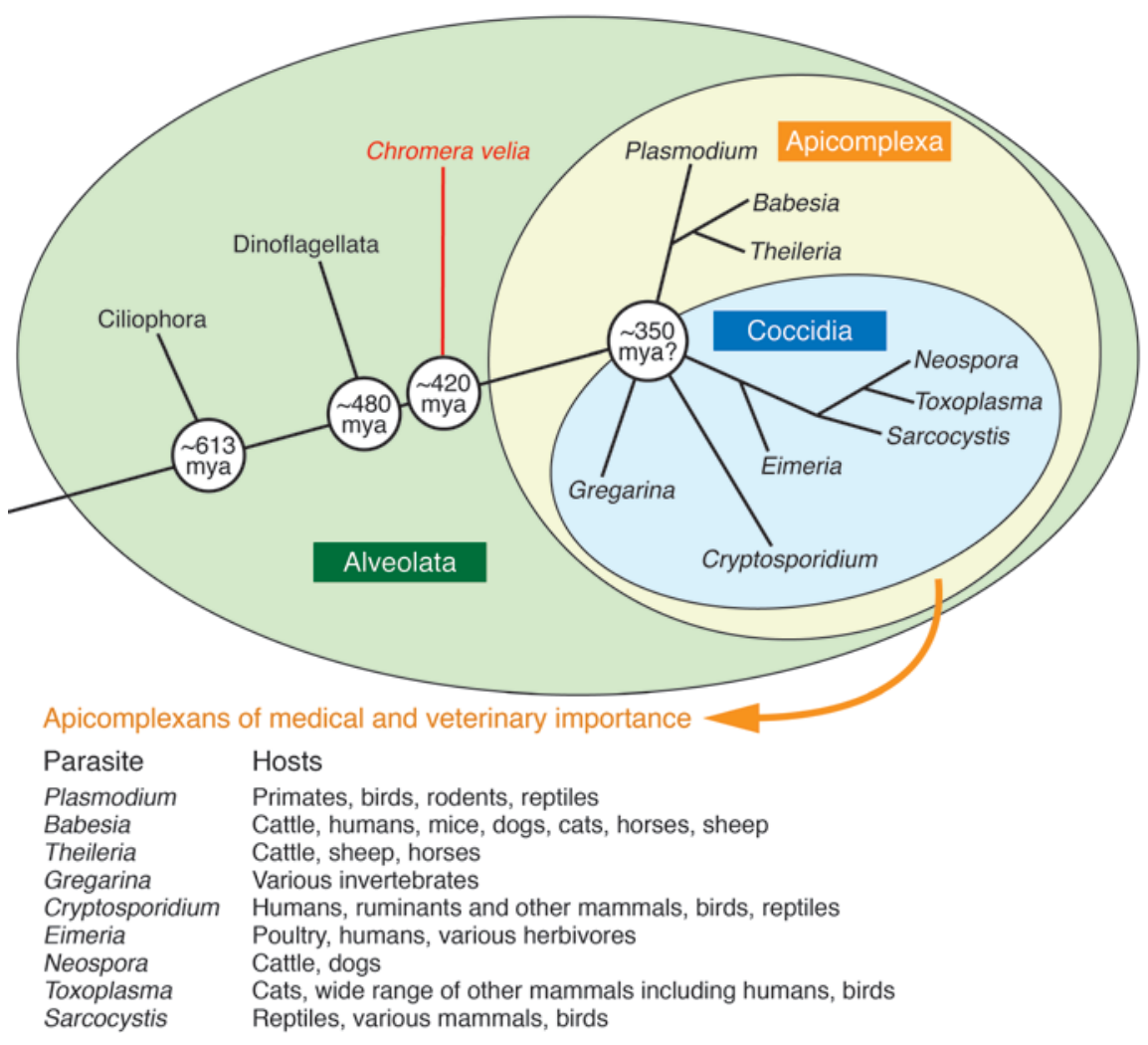

\section{Figure 2}

Apicomplexans and their ancestors. Apicomplexans are obligate intracellular protozoa from ancestors that also gave rise hundreds of millions of years ago (mya) to ciliates, dinoflagellates, and the recently discovered Chromera velia (4). Symbiotic relationships of C. velia and dinoflagellate species with invertebrates suggest that parasitism developed early in the origin of animals. Depicted is an evolutionary tree of the alveolate protozoans, including $C$. velia, a photosynthetic symbiont of corals (4). The phylogeny of Apicomplexa is not fully resolved, and confirmation of the date of the radiation and branch order awaits further studies. Modified with permission from Trends in Parasitology (ref. 119, using information available in refs. 5 and 120). failed to break transmission. Great reductions in the number of malaria cases were nevertheless achieved. More recently, programs providing antimalarial drugs and insecticide-treated bed nets have produced appreciable reductions in malaria and malaria-associated deaths in other African countries, including Zambia, Rwanda, Tanzania, Kenya, The Gambia, and South Africa (18-22).

In view of the major role of An. gambiae as a vector for Plasmodium species, a full genome sequence of An. gambiae, with analyses of its genes and proteins, was reported in 2002 (23). Insights from these data should contribute to future strategies to reduce the intensity of mosquito transmission of malaria-causing parasites. These insights include new findings on An. gambiae systems for sensing hosts, further understanding of which could possibly lead to new interventions to reduce contact between humans and mosquitoes. For example, the identification of odor receptors in the An. gambiae genome and the ability to perform functional studies on orthologs in the Drosophila melanogaster model led to the recent demonstration that the repellent $N, N$-diethyl-meta-toluamide (DEET) inhibits insect odor receptors (24). Other insights may suggest ways to interfere with blood feeding and insect immune defenses (25). Basic research on insecticide resistance and targets for new insecticides may also be transformed by methods such as genome-wide transcriptional analyses and candidate gene-silencing techniques $(25,26)$.

\section{Abundant human genome polymorphisms selected by life-threatening malaria}

Malaria has exerted great evolutionary pressure on the human genome, resulting in the selection of polymorphisms that can dramatically alter susceptibility of children to life-threatening complications of infection. In 1954, Anthony C. Allison provided important evidence that sickle cell hemoglobin trait (i.e., the presence of one $\beta$-globin gene encoding normal hemoglobin, hemoglobin $\mathrm{A}[\mathrm{HbA}]$, and one $\beta$-globin gene encoding sickle-cell hemoglobin $[\mathrm{HbS}])$ was associated with protection against $P$. falciparum malaria in Kenya (27). Since then, investigators have associated protection against $P$. falciparum and $P$. vivax malaria with a variety of human genetic polymorphisms carried by hundreds of millions of people. While many of these polymorphisms affect the host erythrocyte, others may involve effectors of innate and acquired immunity. Here, we discuss some of these polymorphisms as well as recent insights into their proposed mechanisms of protection.

\section{Erythrocyte polymorphisms and protection against severe malaria}

Many of the polymorphisms that protect against severe malaria are found in the genes encoding $\beta$-globin and other molecules of erythrocytes, the host cells in which parasites multiply and set up the pathological interactions that cause disease symptoms. Taking advantage of growing human haplotype maps and SNP databases, genome-wide association studies are presently underway to expand our understanding of these and additional polymorphisms associated with reduced malaria severity in geographically disparate populations (28). Various models have been described to account for protection by glucose- 6 -phosphate dehydrogenase (G6PD) deficiency, $\alpha$-thalassemia, and other conditions such as the hemoglobinopathies caused by the HbS, hemoglobin $\mathrm{C}(\mathrm{HbC})$, or hemoglobin E (HbE) polymorphisms (29-33) (Table 1).

Early models of protection by G6PD deficiency and the hemoglobinopathies often invoked reduced parasite load as the mechanism, even though these models failed to explain important findings from numerous clinical studies performed since the 1950s. In particular, although the polymorphisms underlying $\mathrm{HbS}, \mathrm{HbC}$, 

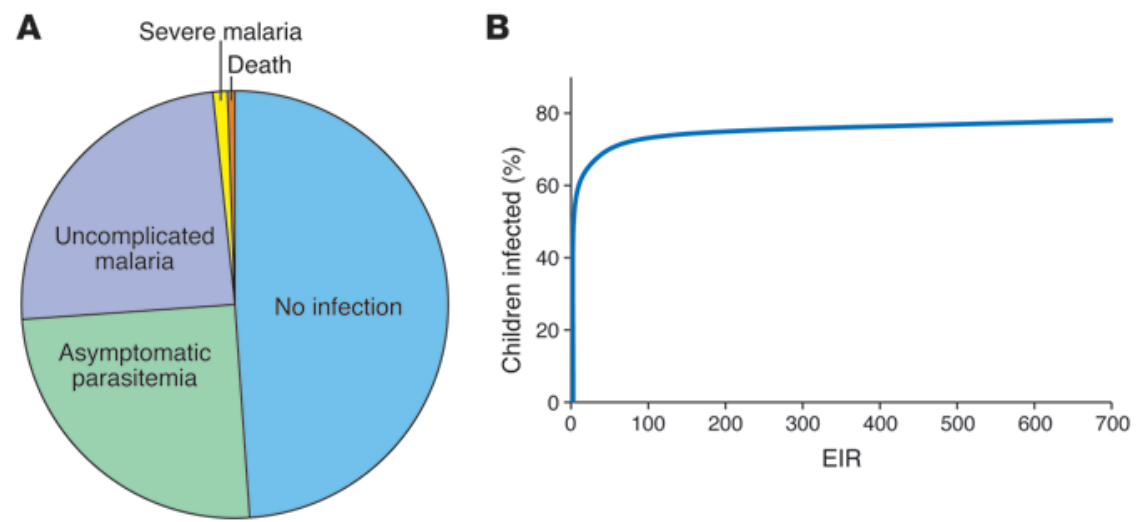

\section{Figure 3}

An. gambiae entomological inoculation rates and infection outcomes in Africa. (A) The clinical outcome of $P$. falciparum sporozoite inoculation by an An. gambiae mosquito depends on many factors and can range from no infection to severe malaria and death. Greenwood et al. (121) estimated that in areas of high transmission, for every 400 infectious bites, 200 result in a parasite infection, half of which will develop uncomplicated malaria, with two cases of severe malaria and one death. (B) Relationship between the mosquito entomological inoculation rate (EIR) and the proportion of individuals infected with $P$. falciparum (14). Analysis of combined data from over 90 African communities indicated that $20 \%$ of people received $80 \%$ of infections and that enormous reductions of existing EIRs would be required to achieve even a modest decrease in parasite prevalence. For example, a two-fold reduction in the EIR, from 200 to 100 infectious bites per year, would reduce parasite prevalence by only $4 \%$, whereas a 100 -fold reduction in the EIR, from 100 infectious bites to 1 infectious bite, would reduce parasite prevalence from about $70 \%$ to $30 \%$. Part B was generated based on data from ref. 14 .

$\alpha$-thalassemia, and G6PD deficiency confer substantial protection against life-threatening malaria, these polymorphisms often confer less protection against uncomplicated malaria and provide little or no resistance to infection itself (34-37). Further, parasitemias in individuals who carry $\mathrm{HbC}$ or have G6PD deficiency show little or no overall difference from parasitemias in control individuals (34, $36,37)$. These observations remained difficult to reconcile with models of protection based on reduction in parasite load, as such reductions predict lower parasitemias and decreased risk of both uncomplicated and severe malaria. Protection that occurs differentially against severe malaria and does not depend on reduction of parasite load requires alternative explanations, one of which we have proposed is at the interface of the parasitized erythrocyte and the microvascular endothelial cell (38).

Various forms of severe $P$. falciparum malaria arise from the sequestration of mature parasitized erythrocytes in the capillaries and microvenules of organs such as the brain, heart, lung, liver, kidney, dermis, and bone marrow. In pregnant women, malaria may cause fetal growth restriction, preterm delivery, or fetal loss because $P$. falciparum-infected erythrocytes accumulate in the proteoglycan matrix of the placental intervillous spaces. By sequestering in organs, mature $P$. falciparum-infected erythrocytes are thought to avoid being

\section{Table 1}

Hemoglobin and erythrocyte polymorphisms protective against life-threatening $P$. falciparum malaria

$\begin{array}{lcc}\text { Name } & \text { Gene affected } & \text { Polymorphism } \\ \mathrm{HbS} & H B B & \beta 6: \text { glutamate to valine }\end{array}$

\section{Proposed mechanisms of protection (Reference) \\ Increased sickling of parasitized erythrocytes, which undergo enhanced clearance by the spleen $(124,125)$}

Reduced erythrocyte invasion and inhibited parasite growth under conditions of low oxygen tension in venous microvessels $(29,126)$

Enhanced phagocytosis of erythrocytes infected with ring-stage parasites (31)

Altered PfEMP-1 display and reduced cytoadherence of parasitized erythrocytes (44)

Enhancement of innate and acquired immunity (57)

$\begin{array}{lcc}\mathrm{HbC} & H B B & \beta 6: \text { glutamate to lysine } \\ \mathrm{HbE} & H B B & \beta 26: \text { glutamate to lysine } \\ & & \\ \alpha \text {-thalassemia } & \text { HBA1/HBA2 } & 3.7-\mathrm{kb} \text { deletion }\end{array}$

Altered PfEMP-1 display and reduced cytoadherence of parasitized erythrocytes (38)

Unidentified membrane abnormality that renders the majority of erythrocytes relatively resistant to invasion (32)

Reduced expression of CR1, a receptor that mediates the binding of noninfected erythrocytes to parasitized erythrocytes (127)

Increased microerythrocyte count in homozygotes reduces the amount of hemoglobin lost for any given parasite density, thus protecting against severe anemia (128) 
A

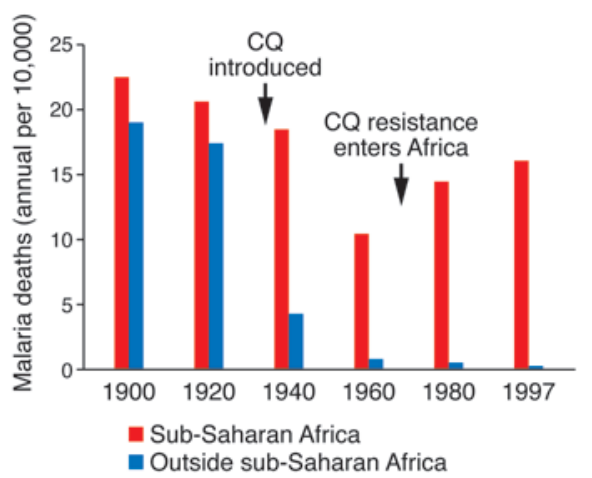

B

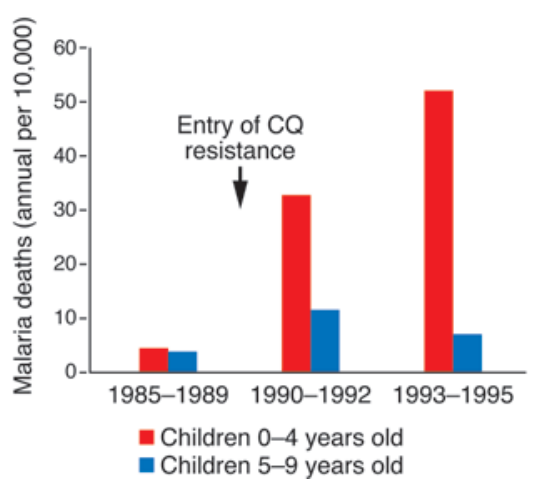

Figure 4

Malaria death rates in Africa fell after the introduction of chloroquine and rose again in the wake of chloroquine-resistant malaria. (A) Malaria death rates in the 20th century. Dramatic reductions in mortality occurred outside sub-Saharan Africa. In Africa, mortality rates declined after the introduction of chloroquine but then rose again with the spread of chloroquine-resistant parasites. (B) Rise in mortality associated with the entry of chloroquine-resistant $P$. falciparum in the village of Mlomp, Senegal. Deaths were primarily among children less than 5 years old, the most susceptible age group in highly malaria-endemic areas. CQ, chloroquine. Part $\mathbf{A}$ was generated based on data from ref. 106. Part B was reproduced with permission from Elsevier (107).

ous forces of natural selection were exerted on these polymorphisms. Oxidative instability and hemoglobin denaturation may underlie the effects of $\mathrm{HbS}$ and $\mathrm{HbC}$ on PfEMP-1 display; these effects may involve elevated levels of membrane-bound hemichromes (oxidized, denatured hemoglobin molecules) binding to the inner aspect of the erythrocyte membrane and interfering with the trafficking and anchoring of PfEMP-1 $(38,44)$.

\section{Polymorphism mitigating placental inflammation in malaria of pregnancy} Inflammation caused by parasitized erythrocytes in the placenta is associated with intrauterine growth restriction, miscarriage, and premature delivery $(47,48)$. Mothers in their first pregnancy are particularly susceptible to these complications, even if they have preexisting immunity as a result of previous $P$. falciparum infection. A substantial part of this susceptibility may be attributed to the expression of PfEMP-1 forms that bind placental chondroitin suldestroyed by the spleen (39). Sequestration can also be compounded by direct binding of noninfected erythrocytes to parasitized erythrocytes (rosetting) and platelet-mediated clumping of parasitized erythrocytes (autoagglutination) $(40,41)$. Inflammatory cells, thrombin deposits, activated tissue factor, upregulated endothelial receptors, and proinflammatory cytokines have all been associated with sequestered P. falciparum-infected erythrocytes and contribute to circulatory impairment, metabolic stress, and tissue compromise in individuals with severe malaria and malaria of pregnancy $(42,43)$.

How might heterozygous states of HbS (sickle-cell trait) or HbC reduce the effects of parasitemia and provide differential protection against severe malaria? In recent studies, the endothelial cell binding of parasitized erythrocytes containing either $\mathrm{HbS}$ or $\mathrm{HbC}$ was found to be reduced relative to the binding of parasitized erythrocytes containing $\mathrm{HbA}(38,44)$. Further, this reduced binding to endothelial cells correlated with decreased surface levels and altered distribution of $P$. falciparum erythrocyte membrane protein- 1 (PfEMP-1), the major variable cytoadherence protein expressed by the parasites on their host erythrocytes $(38,44)$. PfEMP-1 display may therefore be altered sufficiently by these hemoglobinopathies to reduce endothelial cell activation, but not to prevent sequestration, so that a substantial fraction of parasitized erythrocytes still avoid being carried away to the spleen. The benefits of reduced endothelial activation, including reduced upregulation of adhesion molecules, reduced activation of tissue factor and thrombin, decreased amounts of proinflammatory cytokines, and decreased recruitment of platelets and monocytes, would be expected to ameliorate the consequences of local and systemic inflammation.

Molecular-genetic investigations have shown that HbS was selected independently at least five times in Africa, Arabia, and India, that $\mathrm{HbC}$ arose from at least two independent foci in West Africa and Thailand, and that different genetic forms of $\alpha$-thalassemia exist in Asia and Africa $(45,46)$. The independent selection of $\mathrm{HbS}$, despite the deleterious nature of the homozygous HbS state (i.e., sickle-cell disease), provides strong evidence that vigor- fate A (CSA) and are antigenically new to the immune systems of women who have not previously been pregnant (49-51). Less frequent complications in subsequent pregnancies are thought to be explained by acquired immunity to CSA-binding PfEMP-1 molecules $(52,53)$. The importance of placental inflammation in malaria of pregnancy was recently reinforced by data suggesting that polymorphisms affecting expression of the FMS-like tyrosine kinase 1 (FLT1) gene, which encodes a receptor for VEGF (which can act as a proinflammatory cytokine), are under natural selection in this condition. In a study of Tanzanian mothers and infants (54), maternal genotypes of relatively increased FLT1 expression (as a result of homozygosity for short dinucleotide repeat polymorphisms in the $3^{\prime}$ untranslated region [i.e., SS dinucleotide repeat genotypes]) were associated with prior fetal losses in first-time mothers with placental malaria, and SS genotypes in the newborns of first-time mothers were linked to higher occurrences of low birth weight. Placentas delivered with the SS newborns also showed greater inflammation, as judged by histological findings and elevated levels of transcripts encoding IFN- $\gamma$ and immunoglobulin heavy chains. Relatively decreased levels of VEGF-FLT1 signaling associated with genotypes containing long dinucleotide repeat polymorphisms (i.e., SL and LL genotypes) thus might confer increased fetal fitness in utero by reduced maternal inflammatory response to placental $P$. falciparum infection. Interestingly, a soluble form of FLT1 (sFlt1) that binds VEGF and antagonizes FLT1 signaling has been shown to be elevated in preeclampsia (55), raising the possibility of selection by malaria in the evolution of preeclampsia (54).

\section{Variations of innate and acquired immunity modulate malaria susceptibility}

In malaria-endemic areas of Africa, very young children are most susceptible to the severe manifestations of malaria and carry the greatest burden of mortality (56). After about 5 years of age, African children become less susceptible to severe malaria, although 


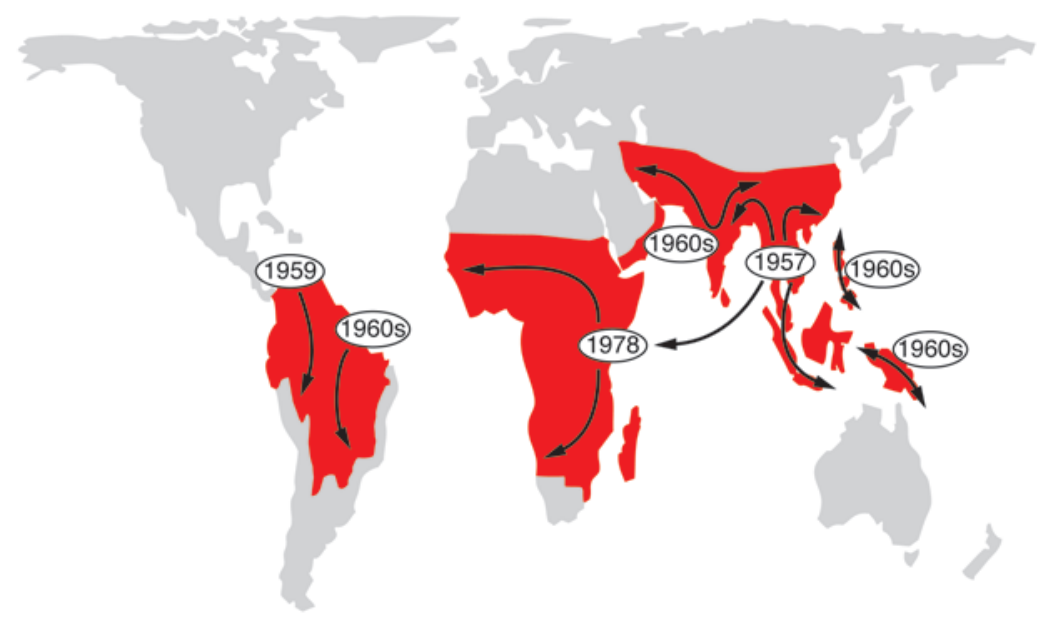

\section{Figure 5}

Chloroquine-resistant $P$. falciparum spread from at least six known origins. Chloroquine resistance entered Africa in the late 1970s, having originated in Southeast Asia. PfCRT molecules from the individual foci all contain a key K76T amino-acid replacement but are distinguished by different patterns of accompanying codon polymorphisms and chromosome haplotype polymorphisms. In addition to five foci previously depicted (109), the map indicates recently described parasites in India and Iran that probably spread from an additional focus of chloroquine resistance (122, 123). Figure reproduced with permission from ASM Press (109). they can continue to suffer episodes of debilitating febrile illness. By adolescence, they can be continually infected with parasites but have only mild symptoms or none at all.

Multiple effectors of the innate and adaptive immune systems are involved in the development of "disease-controlling" immunity, which may be accelerated in the presence of such polymorphisms as those underlying $\mathrm{HbS}$ and $\alpha$-thalassemia $(57,58)$. Enhanced phagocytosis of mutant erythrocytes containing parasites in their early growth stages (ring stages) may also contribute to protection in children with $\mathrm{HbS}$ (31). Variations in the prevalence of severe P. falciparum malaria among ethnic populations of West Africa have been associated with polymorphisms in the interferon regulatory factor- 1 gene and a functional deficit of Tregs (59-61). While the role of Treg cells in malaria protection is unknown, they have been shown to affect in vitro $T$ cell proliferative responses to $P$. falciparum antigens (61).

A growing body of evidence suggests that TNF activity may be involved in the immune control and disease manifestations of P. falciparum malaria. Infected erythrocytes may induce TNF release from monocytes through cytoadherence interactions or by the release of parasite-derived factors. For example, $P$. falciparum glycosylphosphatidylinositol, which is released upon schizont rupture, signals through TLR2 and TLR4 on monocytes to induce TNF production (62). While TNF is believed to help control parasitemia, it also induces fever during malaria episodes and may aggravate cerebral malaria by upregulating the expression of ICAM-1 and other leukocyte adhesion receptors on brain microvascular endothelial cells (63). TNF levels are highest in children with severe malaria and fatal outcomes $(64,65)$.

Gene promoter polymorphisms that increase TNF expression (66, $67)$ accelerate parasite clearance and symptom resolution $(68,69)$; they can also enhance the inflammatory consequences of infection and susceptibility to severe malaria and death $(70,71)$. TNF production during malaria episodes may also be up- or downregulated by polymorphisms that either alter the signal-transducing domains of TLR2 and TLR4 or modify the function of components of their downstream signaling cascades. Common TLR4 polymorphisms (encoding Asp299Gly and Thr399Ile substitutions) have been associated in African populations with severe malaria in children (72) and with maternal anemia and the delivery of low-birth-weight newborns (73), but the effects of these polymorphisms on TLR4 function are not known. TLR1 and TLR6 polymorphisms have also been associated in Brazilian populations with the development of uncomplicated malaria (74). Another polymorphism in the gene encoding MyD88 adapter-like protein (MAL; also known as TIRAP), the adaptor protein that conveys TLR2- and TLR4-initiated signaling events, has been associated not only with protection against malaria, but also with protection against pneumonia, sepsis, and tuberculosis (75). This MAL polymorphism attenuates TLR2-mediated signaling, suggesting that several TLR polymorphisms might have also evolved to dampen the production of TNF during episodes of malaria.

The mechanisms by which individuals acquire immunity to malaria are not well understood. Although T cell-and antibody-mediated responses develop against a number of parasite antigens during infection, no immune response has been found to be superior to patient age and previous parasite exposure as a correlate of protective immunity. In an example of the importance of age-associated protective immunity, older children in Mali treated with chloroquine were better able to clear chloroquine-resistant $P$. falciparum parasites than their younger counterparts (76). The influence of previous parasite exposure is evident in a retrospective analysis of 1940-1963 data from patients who were infected with $P$. falciparum to treat their tertiary syphilis (the parasites produced what were believed to be curative fever spikes) (77). The infections featured declining parasitemias in successive waves, characteristic of sequential populations of parasites expressing different surface antigens on their host erythrocytes (see the following section). Furthermore, when individuals who cleared an infection were reinfected by either the same or a different $P$. falciparum strain, most developed parasitemia and fever - although the fever tended to be less intense and parasitemia tended to be lower than during the initial infection $(78,79)$. These and related findings from the literature (80-82) suggest that prior experience with malaria leads to the development of immunity that controls the severity of disease rather than that which prevents the development of symptoms or appreciably dense parasitemias.

\section{Diverse mechanisms for immune system evasion and erythrocyte invasion}

Antigen switching - a process by which P. falciparum parasite populations change expression from one PfEMP-1 variant-encoding gene (var gene) to another - creates distinct waves of parasitemia that must be chased by different antibody responses in the course of bloodstream infection. These waves, along with those produced from additional P. falciparum infections, can cause recurrent fevers that may not be cleared for many months or years but are impor- 
tant components of the malaria experience that leads to eventual acquisition of disease-controlling immunity. Field studies have associated this immunity with the ability of serum to recognize the diverse PfEMP-1 molecules of heterologous parasite strains. As children grow and expand their antibody repertoire (83-85), they become susceptible to fewer and fewer $P$. falciparum strains. Genome and expression analyses have shown that the PfEMP-1 family has a vast capacity for sequence diversification $(86,87)$, and some PfEMP-1 variants may cause more severe clinical outcomes than others $(88,89)$. Structural constraints on the extracellularbinding domains of PfEMP-1 may restrain the ability of different variants to escape recognition by cross-reactive antibodies, thus allowing some degree of protection between strains $(90,91)$.

A primary determinant of host specificity and virulence for all malaria-causing parasites is their ability to invade the host erythrocyte. P. falciparum, perhaps more than any other Plasmodium parasite of humans, uses a variety of ligand-receptor interactions to invade erythrocytes through redundant or alternative pathways $(92,93)$. Two families of $P$. falciparum ligands illustrate this adaptability: first, $P$. falciparum erythrocyte-binding antigen 175 (PfEBA-175) and its paralogs in the family of PfEBA and $P$. falciparum erythrocytebinding ligand (PfEBL) proteins, including PfEBA-140 (also known as BAEBL), PfEBA-181 (also known as JESEBL), and PfEBL-1 (also known as EBA-1); and second, proteins that, based on their homology to $P$. vivax reticulocyte-binding proteins, are termed $P$. falciparum reticulocyte-binding protein homologs (PfRH) and include PfRH1, PfRH2a, PfRH2b, PfRH4, and PfRH5 (93). Several members of these families are sialic acid-dependent, which means they bind neuraminidase-sensitive receptors on erythrocytes, whereas other members are considered sialic acid-independent because they can bind to erythrocytes that are either deficient in various glycophorins or stripped of their sialic residues by neuraminidase (92). Point polymorphisms in the genes encoding PfEBA-181 and PfEBA-140 modulate receptor binding and may change the specificities of these proteins for different receptors on human erythrocytes (94-96). In the PfRH family of ligands, upregulation of PfRH4 has been linked through microarray and transfection studies to the ability of a $P$. falciparum line to switch from sialic acid-dependent to sialic acid-independent invasion $(97,98)$. More recently, a point polymorphism in the gene encoding PfRH5 was linked to the ability of a $P$. falciparum line to infect the erythrocytes of Aotus nancymaae monkeys and cause severe malaria in these primates (99). PfRH5 molecules containing this polymorphism bound to a neuraminidase-sensitive receptor on Aotus and human erythrocytes, whereas PfRH5 from a parasite that could not infect Aotus bound to no receptor on the monkey erythrocytes and to a neuraminidase-resistant receptor on human erythrocytes (99). Invasion pathways can thus be determined by the erythrocyte surface receptors to which PfRH family members bind, in contrast with a recent proposal that $\mathrm{PfRH}$ ligands must interact with various PfEBLs to determine invasion pathway specificity (8).

$P$. vivax stands apart from $P$. falciparum in its lack of a family of proteins analogous to PfEMP-1 and its restriction to reticulocytes expressing Duffy blood group antigens. These observations imply mechanisms of survival in the host that are significantly different from those used by P. falciparum. P. vivax does not depend on sequestering for survival and is therefore usually not associated with microvascular obstruction and regional inflammation, two pathological features of $P$. falciparum malaria. Rather, mature $P$. vivax parasites are thought to circulate through the spleen and avoid destruction by other means, possibly by mechanisms that include increased (rather than decreased) deformability of the infected host erythrocytes (100). The restriction of $P$. vivax to reticulocytes appears to involve a family of binding proteins (PvRBPs) that target surface receptors of these host cells $(8,101)$. This restriction is in addition to Duffy blood group-antigen selectivity. The high prevalence of individuals who have lost Duffy blood group antigen from their erythrocytes accounts for the rarity of P. vivax in West sub-Saharan Africa (102). An independent origin of Duffy blood group antigen-negativity has been found in Papua New Guinea (103), where the heterozygous state is associated with reduced prevalence of $P$. vivax infection (104).

The genome sequence information that is now available from $P$. falciparum, P. vivax, and P. knowlesi opens opportunities to understand the many contrasting features of erythrocyte invasion, pathogenesis, and immune system evasion associated with these species (8-10). Only P. vivax shows strong restriction to reticulocytes. $P$. vivax and $P$. knowlesi, but not P. falciparum, utilize the Duffy blood group antigen for erythrocyte invasion. While the var-encoded PfEMP-1 antigens of $P$. falciparum share some characteristics with P. knowlesi SICAvar antigens (105), no antigen family with similar characteristics has been identified in P. vivax (8). On the other hand, the P. vivax vir and $P$. knowlesi kir members of the Plasmodium interspersed repeats (pir) gene superfamily have features that show closer relationships to each other than to the more distantly related $P$. falciparum surfin and $P f m c-2 t m$ families of genes (8). Much work remains to clarify the roles of these and other multicopy gene families.

\section{The fall of chloroquine and rise of artemisinins}

The introduction of the antimalarial chloroquine and the pesticide dichloro-diphenyl-trichloroethane (DDT) toward the end of World War II brought dramatic new power to malaria control programs (15). Optimism ran high that these chemicals could be used to eliminate malaria, and in 1955 , the WHO launched a campaign to eradicate the disease. While that goal proved unattainable, the campaign achieved regional successes and reduced malaria incidence in many areas of the world. In Africa, where the enormity of An. gambiae transmission and futility of large-scale DDT-spraying programs were recognized, chloroquine was so cheap, effective, and easy to use that it soon was provided everywhere in massive quantities. Death rates from malaria among children in Africa dropped and, in the 1970s, approached half the level of the prechloroquine years (Figure 4A) (106). However, chloroquine-resistant $P$. falciparum entered Africa in the late 1970 s and began to spread rapidly under drug pressure. Morbidity and mortality consequently resurged (107) (Figure 4); in the late 1990s, death rates from malaria across large areas were again approaching the levels of prechloroquine years.

The former efficacy of chloroquine against $P$. falciparum created one of the greatest global health impacts of any antimicrobial drug ever discovered. As resistant parasites spread from their original foci across malaria-endemic regions (Figure 5), treatment failures provided a clear demonstration of how easily a drug can be rendered ineffective when tremendous numbers of pathogens with rapid reproduction cycles are put under widespread selection pressure. Chloroquine-resistant $P$. falciparum is one of many examples in a growing list of drug-resistant organisms that have evolved within the short span of just a couple of human generations, including multidrug-resistant Mycobacterium tuberculosis and other highly antibiotic-resistant bacteria (108) that increasingly threaten our world's communities and hospitals. 
Genetic linkage studies have shown that chloroquine resistance in P. falciparum is determined by polymorphisms in the gene encoding a molecule termed PfCRT, a transporter that, in modified form, promotes drug efflux from parasite digestive vacuoles (reviewed in ref. 109). Strains of P. vivax that are highly resistant to chloroquine have been documented in recent years in Oceania and parts of Asia, and cases of P. vivax malaria unresponsive to chloroquine treatment have also been reported in South America; but these forms of resistance seem to involve a mechanism independent of polymorphisms in a P. vivax ortholog of PfCRT (110). Resolving the mechanism of $P$. vivax chloroquine resistance will require fresh investigative approaches, as many of the critical laboratory culture and genetic methods available for $P$. falciparum are not available for $P$. vivax. Mapping a critical locus of the resistance mechanism may be possible through the use of genome-sequence data and SNPs in genome-wide surveys of $P$. vivax samples that differ in drug responses.

The problem of chloroquine resistance emphasizes the necessity of discovering new replacement drugs through research. It also emphasizes the importance of preserving current antimalarial drugs by effective stewardship, assurance of open and up-to-date sources of information, appropriate education, and use of antimalarial drugs in combinations, rather than as monotherapies that are more prone to select resistant strains. Effective control of antimalarials - including halting their counterfeit and black market distributions - in some cases can promote the return of drug-sensitive parasite strains to regions that have overwhelming levels of drug-resistant malaria. Recent studies from Malawi showed that a policy that removed chloroquine as an available treatment in 1993 was followed by repopulation of the region with chloroquine-sensitive strains by 2001 (111). A similar but slower return of chloroquine-sensitive strains has also been observed in Hainan, People's Republic of China, where use of the drug against P. falciparum malaria was suspended in 1979 (112).

Today, artemisinin-based combination therapies (ACTs) are the leading replacements for chloroquine, but costs to consumers must still be reduced if these drug combinations are to be as available and affordable as chloroquine once was. The artemisinin derivatives, discovered and developed in the 1970s by research teams in the People's Republic of China (113), result in rapid parasite clearance and clinical responses, making them especially valuable for the treatment of severe malaria. All artemisinin derivatives remain effective in Southeast Asia, although there are worrisome concerns about delayed parasite clearance times in areas of Cambodia and Thailand $(114,115)$. It is therefore critical to protect the artemisinins by stopping monotherapy and using them only in combination with effective partner drugs in ACTs. Unfortunately, parasite strains are often already resistant to partner drugs (e.g., lumefantrine, amodiaquine, mefloquine, and sulfadoxine-pyrimethamine) in the first generation ACTs recommended by the WHO. Second generation ACTs con- taining more effective partner drugs (e.g., piperaquine) are now being developed and deployed.

\section{Perspective}

Global action against malaria is receiving great boosts from new coalitions and funding, notably reflected in the Global Malaria Action Plan created in 2008 by the Roll Back Malaria partnership and backed by the United Nations (http://www.rollbackmalaria. org/gmap/). Widespread deployment of readily available ACTs, long-lasting insecticide-treated bed nets, indoor residual spraying with insecticides, and programs of intermittent presumptive therapy that reduce the number of malaria episodes in children and pregnant women will have important effects on the burden of disease in malarious regions. Indeed, in results reminiscent of the impact of chloroquine across Africa in the 1960s and 1970s, today's improved access to drug combination treatments and long-lasting insecticide-treated bed nets is producing appreciable reductions in deaths from malaria in a number of African countries. As long as resistance to ACTs and insecticides remains in check, renewed efforts with current tools should be able to provide better than the approximately $50 \%$ reduction of mortality achieved in the period of chloroquine's greatest efficacy. Drug and insecticide resistance are bound to spread, however, and discoveries from a solid foundation of biological understanding will be vital to effective malaria control in the future.

Beyond current efforts to reduce the burden of disease, the much more difficult battles to sustain these reductions and eliminate transmission will require taking on the vectorial capacities of mosquitoes such as An. gambiae and the immense source of highly adaptable parasites they transmit from asymptomatic individuals. Success in these battles will be achieved only by lasting breaks in the links of parasite transmission between humans and mosquitoes. While new technologies such as antisporozoite vaccines (116) promise to be useful in this regard, it is clear that much more than technological innovation is required. Malaria promotes poverty, and poverty promotes malaria (117). Lasting advances against malaria transmission will be rooted in the economic, educational, and social advances that lift populations out of poverty, empower their communities for malaria control, and foster improvements in housing, environment, and health infrastructure that close out opportunities for the parasites to thrive.

\section{Acknowledgments}

The authors are supported by the Intramural Research Program of the National Institute of Allergy and Infectious Diseases.

Address correspondence to: Thomas E. Wellems, Laboratory of Malaria and Vector Research, Twinbrook III Building, Room 3E10D, National Institute of Allergy and Infectious Diseases, NIH, Bethesda, Maryland 20892-8132, USA. Phone: (301) 496-4021; Fax: (301) 402-2201; E-mail: twellems@niaid.nih.gov.

\footnotetext{
1. Snow, R.W., Guerra, C.A., Noor, A.M., Myint, H.Y., and Hay, S.I. 2005. The global distribution of clinical episodes of Plasmodium falciparum malaria. Nature. 434:214-217.

2. WHO. 2008. World malaria report. World Health Organization Press. Geneva, Switzerland. http:// apps.who.int/malaria/wmr2008/malaria2008.pdf.

3. Cox-Singh, J., et al. 2008. Plasmodium knowlesi malaria in humans is widely distributed and potentially life threatening. Clin. Infect. Dis. 46:165-171.
}

4. Moore, R.B., et al. 2008. A photosynthetic alveolate closely related to apicomplexan parasites. Nature. 451:959-963.

5. Okamoto, N., and McFadden, G.I. 2008. The mother of all parasites. Future Microbiol. 3:391-395.

6. Garnham, P.C.C. 1966. Malaria parasites and other haemosporidia. Blackwell Scientific Publications. Oxford, United Kingdom. 1114 pp.

7. Mu, J., et al. 2005. Host switch leads to emergence of Plasmodium vivax malaria in humans. Mol. Biol.
Evol. 22:1686-1693.

8. Carlton, J.M., et al. 2008. Comparative genomics of the neglected human malaria parasite Plasmodium vivax. Nature. 455:757-763.

9. Gardner, M.J., et al. 2002. Genome sequence of the human malaria parasite Plasmodium falciparum. Nature. 419:498-511.

10. Pain, A., et al. 2008. The genome of the simian and human malaria parasite Plasmodium knowlesi. Nature. 455:799-803. 
11. Marsh, K., et al. 1995. Indicators of life-threatening malaria in African children. N. Engl. J. Med. 332:1399-1404.

12. Rowe, A.K., et al. 2006. The burden of malaria mortality among African children in the year 2000. Int J. Epidemiol. 35:691-704.

13. Miller, L.H. 1992. The challenge of malaria. Science. 257:36-37.

14. Smith, D.L., Dushoff, J., Snow, R.W., and Hay, S.I. 2005. The entomological inoculation rate and Plas modium falciparum infection in African children. Nature. 438:492-495.

15. Bruce-Chwatt, L.J. 1993. Essential malariology. Oxford University Press. New York, New York, USA. 384 pp.

16. Soper, F.L., and Wilson, D.B. 1943. Anopheles gambiae in Brazil 1930 to 1940. The Rockefeller Foundation. New York, New York, USA. 262 pp.

17. Molyneux, L., and Grammicia, G. 1980. The Garki Project. Research on the epidemiology and control of malaria in the Sudan savanna of West Africa. World Health Organization Press. Geneva, Switzerland. $311 \mathrm{pp}$.

18. Barnes, K.I., et al. 2005. Effect of artemether-lumefantrine policy and improved vector control on malaria burden in KwaZulu-Natal, South Africa. PLoS Med. 2:e330.

19. Bhattarai, A., et al. 2007. Impact of artemisinin-based combination therapy and insecticide-treated nets on malaria burden in Zanzibar. PLoS Med. 4:e309.

20. Ceesay, S.J., et al. 2008. Changes in malaria indices between 1999 and 2007 in The Gambia: a retrospective analysis. Lancet. 372:1545-1554.

21. Sievers, A.C., et al. 2008. Reduced paediatric hospitalizations for malaria and febrile illness patterns following implementation of community-based malaria control programme in rural Rwanda. Malar. J. 7:167.

22. Steketee, R.W., et al. 2008. National malaria control and scaling up for impact: the Zambia experience through 2006. Am. J. Trop. Med. Hyg. 79:45-52.

23. Holt, R.A., et al. 2002. The genome sequence of the malaria mosquito Anopheles gambiae. Science. 298:129-149.

24. Ditzen, M., Pellegrino, M., and Vosshall, L.B. 2008. Insect odorant receptors are molecular targets of the insect repellent DEET. Science. 319:1838-1842.

25. Blandin, S.A., Marois, E., and Levashina, E.A. 2008. Antimalarial responses in Anopheles gambiae: from a complement-like protein to a complement-like pathway. Cell Host Microbe. 3:364-374.

26. Dimopoulos, G., et al. 2002. Genome expression analysis of Anopheles gambiae: responses to injury, bacterial challenge, and malaria infection. Proc Natl. Acad. Sci. U. S. A. 99:8814-8819.

27. Allison, A.C. 1954. Protection afforded by sicklecell trait against subtertian malareal infection. $\mathrm{Br}$ Med. J. 1:290-294.

28. Jallow, M., et al. 2009. Genome-wide and fineresolution association analysis of malaria in Wes Africa. Nat. Genet. 41:657-665.

29. Pasvol, G., Weatherall, D.J., and Wilson, R.J. 1978. Cellular mechanism for the protective effect of haemoglobin S against P. falciparum malaria. Nature. 274:701-703

30. Cortes, A., Benet, A., Cooke, B.M., Barnwell, J.W. and Reeder, J.C. 2004. Ability of Plasmodium falci parum to invade Southeast Asian ovalocytes varies between parasite lines. Blood. 104:2961-2966.

31. Ayi, K., Turrini, F., Piga, A., and Arese, P. 2004. Enhanced phagocytosis of ring-parasitized mutant erythrocytes: a common mechanism that may explain protection against falciparum malaria in sickle trait and beta-thalassemia trait. Blood. 104:3364-3371.

32. Chotivanich, K., et al. 2002. Hemoglobin E: a balanced polymorphism protective against high parasitemias and thus severe $P$. falciparum malaria.
Blood. 100:1172-1176.

33. Cappadoro, M., et al. 1998. Early phagocytosis of glucose-6-phosphate dehydrogenase (G6PD)-deficient erythrocytes parasitized by Plasmodium falciparum may explain malaria protection in G6PD deficiency. Blood. 92:2527-2534.

34. Guindo, A., Fairhurst, R.M., Doumbo, O.K., Wellems, T.E., and Diallo, D.A. 2007. X-linked G6PD deficiency protects hemizygous males but not heterozygous females against severe malaria. PLoS Med. 4:e66.

35. Ruwende, C., et al. 1995. Natural selection of hemiand heterozygotes for G6PD deficiency in Africa by resistance to severe malaria. Nature. 376:246-249.

36. Modiano, D., et al. 2001. Haemoglobin C protects against clinical Plasmodium falciparum malaria. Nature. 414:305-308.

37. Agarwal, A., et al. 2000. Hemoglobin C associated with protection from severe malaria in the Dogon of Mali, a West African population with a low prevalence of hemoglobin S. Blood. 96:2358-2363.

38. Fairhurst, R.M., et al. 2005. Abnormal display of PfEMP-1 on erythrocytes carrying haemoglobin C may protect against malaria. Nature. 435:1117-1121.

39. David, P.H., Hommel, M., Miller, L.H., Udeinya, I.J., and Oligino, L.D. 1983. Parasite sequestration in Plasmodium falciparum malaria: spleen and antibody modulation of cytoadherence of infected erythrocytes. Proc. Natl. Acad. Sci. U. S. A. 80:5075-5079.

40. Rowe, A., Obeiro, J., Newbold, C.I., and Marsh, K. 1995. Plasmodium falciparum rosetting is associated with malaria severity in Kenya. Infect. Immun. 63:2323-2326.

41. Pain, A., et al. 2001. Platelet-mediated clumping of Plasmodium falciparum-infected erythrocytes is a common adhesive phenotype and is associated with severe malaria. Proc. Natl. Acad. Sci. U. S. A. 98:1805-1810

42. Francischetti, I.M., Seydel, K.B., and Monteiro, R.Q. 2008. Blood coagulation, inflammation, and malaria. Microcirculation. 15:81-107.

43. Dondorp, A.M., et al. 2008. Direct in vivo assessment of microcirculatory dysfunction in severe falciparum malaria. J. Infect. Dis. 197:79-84.

44. Cholera, R., et al. 2008. Impaired cytoadherence of Plasmodium falciparum-infected erythrocytes containing sickle hemoglobin. Proc. Natl. Acad. Sci. U. S. A 105:991-996.

45. Flint, J., Harding, R.M., Boyce, A.J., and Clegg, J.B. 1998. The population genetics of the haemoglobinopathies. Baillieres Clin. Haematol. 11:1-51.

46. Sanchaisuriya, K., et al. 2001. Molecular characterization of hemoglobin C in Thailand. Am.J. Hematol. 67:189-193.

47. Duffy, P.E. 2007. Plasmodium in the placenta: parasites, parity, protection, prevention and possibly preeclampsia. Parasitology. 134:1877-1881.

48. Rogerson, S.J., and Boeuf, P. 2007. New approaches to pathogenesis of malaria in pregnancy. Parasitology. 134:1883-1893.

49. Fried, M., and Duffy, P.E. 1996. Adherence of Plasmodium falciparum to chondroitin sulfate $\mathrm{A}$ in the human placenta. Science. 272:1502-1504.

50. Khattab, A., Kun, J., Deloron, P., Kremsner, P.G., and Klinkert, M.Q. 2001. Variants of Plasmodium falciparum erythrocyte membrane protein 1 expressed by different placental parasites are closely related and adhere to chondroitin sulfate A. J. Infect. Dis. 183:1165-1169.

51. Gamain, B., et al. 2004. Identification of a $67-$ amino-acid region of the Plasmodium falciparum variant surface antigen that binds chondroitin sulphate $A$ and elicits antibodies reactive with the surface of placental isolates. Mol. Microbiol. 53:445-455.

52. Duffy, P.E., and Fried, M. 1999. Malaria during pregnancy: parasites, antibodies and chondroitin sulphate A. Biochem. Soc. Trans. 27:478-482.

53. Duffy, P.E., and Fried, M. 2003. Antibodies that inhibit Plasmodium falciparum adhesion to chondroitin sulfate A are associated with increased birth weight and the gestational age of newborns. Infect. Immun. 71:6620-6623.

54. Muehlenbachs, A., Fried, M., Lachowitzer, J., Mutabingwa, T.K., and Duffy, P.E. 2008. Natural selection of FLT1 alleles and their association with malaria resistance in utero. Proc. Natl. Acad. Sci. U. S. A. 105:14488-14491.

55. Maynard, S.E., et al. 2003. Excess placental soluble fms-like tyrosine kinase 1 (sFlt1) may contribute to endothelial dysfunction, hypertension, and proteinuria in preeclampsia. J. Clin. Invest. 111:649-658.

56. Langhorne, J., Ndungu, F.M., Sponaas, A.M., and Marsh, K. 2008. Immunity to malaria: more questions than answers. Nat. Immunol. 9:725-732.

57. Williams, T.N., et al. 2005. An immune basis for malaria protection by the sickle cell trait. PLoS Med. 2:e128.

58. Williams, T.N., et al. 1996. High incidence of malaria in alpha-thalassaemic children. Nature. 383:522-525

59. Mangano, V.D., et al. 2008. Interferon regulatory factor-1 polymorphisms are associated with the control of Plasmodium falciparum infection. Genes Immun. 9:122-129.

60. Modiano, D., et al. 1996. Different response to Plasmodium falciparum malaria in west African sympatric ethnic groups. Proc. Natl. Acad. Sci. U. S. A. 93:13206-13211.

61. Torcia, M.G., et al. 2008. Functional deficit of T regulatory cells in Fulani, an ethnic group with low susceptibility to Plasmodium falciparum malaria. Proc. Natl. Acad. Sci. U. S. A. 105:646-651.

62. Krishnegowda, G., et al. 2005. Induction of proinflammatory responses in macrophages by the glycosylphosphatidylinositols of Plasmodium falciparum: cell signaling receptors, glycosylphosphatidylinositol (GPI) structural requirement, and regulation of GPI activity. J. Biol. Chem. 280:8606-8616.

63. Armah, H., et al. 2005. Cytokines and adhesion molecules expression in the brain in human cerebral malaria. Int. J. Environ. Res. Public Health. 2:123-131.

64. Awandare, G.A., et al. 2006. Increased levels of inflammatory mediators in children with severe Plasmodium falciparum malaria with respiratory distress. J. Infect. Dis. 194:1438-1446.

65. Day, N.P., et al. 1999. The prognostic and pathophysiologic role of pro- and antiinflammatory cytokines in severe malaria. J. Infect. Dis. 180:1288-1297.

66. Abraham, L.J., and Kroeger, K.M. 1999. Impact of the -308 TNF promoter polymorphism on the transcriptional regulation of the TNF gene: relevance to disease. J. Leukoc. Biol. 66:562-566.

67. May, J., Lell, B., Luty, A.J., Meyer, C.G., and Kremsner, P.G. 2000. Plasma interleukin-10:Tumor necrosis factor (TNF)-alpha ratio is associated with TNF promoter variants and predicts malarial complications. J. Infect. Dis. 182:1570-1573.

68. Mordmuller, B.G., et al. 1997. Tumor necrosis factor in Plasmodium falciparum malaria: high plasma level is associated with fever, but high production capacity is associated with rapid fever clearance. Eur. Cytokine Netw. 8:29-35.

69. Kremsner, P.G., et al. 1995. Prediction of accelerated cure in Plasmodium falciparum malaria by the elevated capacity of tumor necrosis factor production. Am. J. Trop. Med. Hyg. 53:532-538.

70. McGuire, W., Hill, A.V., Allsopp, C.E., Greenwood, B.M., and Kwiatkowski, D. 1994. Variation in the TNF-alpha promoter region associated with susceptibility to cerebral malaria. Nature. 371:508-510.

71. McGuire, W., et al. 1999. Severe malarial anemia and cerebral malaria are associated with different tumor necrosis factor promoter alleles. J. Infect. Dis. 179:287-290

72. Mockenhaupt, F.P., et al. 2006. Toll-like receptor (TLR) polymorphisms in African children: Com- 
mon TLR-4 variants predispose to severe malaria. Proc. Natl. Acad. Sci. U. S. A. 103:177-182.

73. Mockenhaupt, F.P., et al. 2006. Common polymorphisms of toll-like receptors 4 and 9 are associated with the clinical manifestation of malaria during pregnancy. J. Infect. Dis. 194:184-188.

74. Leoratti, F.M., et al. 2008. Variants in the toll-like receptor signaling pathway and clinical outcomes of malaria. J. Infect. Dis. 198:772-780.

75. Khor, C.C., et al. 2007. A Mal functional variant is associated with protection against invasive pneumococcal disease, bacteremia, malaria and tuberculosis. Nat. Genet. 39:523-528.

76. Djimde, A.A., et al. 2003. Clearance of drug-resistant parasites as a model for protective immunity in Plasmodium falciparum malaria. Am. J. Trop. Med. Hyg. 69:558-563.

77. Collins, W.E., and Jeffery, G.M. 1999. A retrospective examination of sporozoite- and trophozoite-induced infections with Plasmodium falciparum: development of parasitologic and clinical immunity during primary infection. Am. J. Trop. Med. Hyg. 61:4-19.

78. Collins, W.E., and Jeffery, G.M. 1999. A retrospective examination of secondary sporozoite- and trophozoite-induced infections with Plasmodium falciparum: development of parasitologic and clinical immunity following secondary infection. Am.J. Trop. Med. Hyg. 61:20-35.

79. Collins, W.E., and Jeffery, G.M. 1999. A retrospective examination of sporozoite- and trophozoiteinduced infections with Plasmodium falciparum in patients previously infected with heterologous species of Plasmodium: effect on development of parasitologic and clinical immunity. Am. J. Trop. Med. Hyg. 61:36-43.

80. Gupta, S., Snow, R.W., Donnelly, C.A., Marsh, K., and Newbold, C. 1999. Immunity to non-cerebral severe malaria is acquired after one or two infections. Nat. Med. 5:340-343.

81. Hviid, L. 2005. Naturally acquired immunity to Plasmodium falciparum malaria in Africa. Acta Trop. 95:270-275.

82. Doolan, D.L., Dobano, C., and Baird, J.K. 2009. Acquired immunity to malaria. Clin. Microbiol. Rev. 22:13-36.

83. Bull, P.C., et al. 2000. Plasmodium falciparum-infected erythrocytes: agglutination by diverse Kenyan plasma is associated with severe disease and young host age. J. Infect. Dis. 182:252-259.

84. Ofori, M.F., et al. 2002. Malaria-induced acquisition of antibodies to Plasmodium falciparum variant surface antigens. Infect. Immun. 70:2982-2988.

85. Bull, P.C., et al. 1998. Parasite antigens on the infected red cell surface are targets for naturally acquired immunity to malaria. Nat. Med. 4:358-360.

86. Frank, M., et al. 2008. Frequent recombination events generate diversity within the multi-copy variant antigen gene families of Plasmodium falciparum. Int. J. Parasitol. 38:1099-1109.

87. Trimnell, A.R., et al. 2006. Global genetic diversity and evolution of var genes associated with placental and severe childhood malaria. Mol. Biochem. Parasitol. 148:169-180.

88. Normark, J., et al. 2007. PfEMP1-DBL1alpha amino acid motifs in severe disease states of Plasmodium falciparum malaria. Proc. Natl. Acad. Sci. U. S. A. 104:15835-15840.

89. Kaestli, M., et al. 2006. Virulence of malaria is associated with differential expression of Plasmodium falciparum var gene subgroups in a case-control study. J. Infect. Dis. 193:1567-1574.

90. Mackintosh, C.L., et al. 2008. Acquisition of naturally occurring antibody responses to recombinant protein domains of Plasmodium falciparum erythrocyte membrane protein 1. Malar. J. 7:155.

91. Bull, P.C., et al. 2008. Plasmodium falciparum antigenic variation. Mapping mosaic var gene sequences onto a network of shared, highly polymorphic sequence blocks. Mol. Microbiol. 68:1519-1534.

92. Hadley, T.J., et al. 1987. Falciparum malaria parasites invade erythrocytes that lack glycophorin A and $\mathrm{B}(\mathrm{MkMk})$. Strain differences indicate receptor heterogeneity and two pathways for invasion. J. Clin. Invest 80:1190-1193.

93. Iyer, J., Gruner, A.C., Renia, L., Snounou, G., and Preiser, P.R. 2007. Invasion of host cells by malaria parasites: a tale of two protein families. Mol. Microbiol. 65:231-249.

94. Mayer, D.C., Mu, J.B., Feng, X., Su, X.Z., and Miller, L.H. 2002. Polymorphism in a Plasmodium falciparum erythrocyte-binding ligand changes its receptor specificity. J. Exp. Med. 196:1523-1528.

95. Mayer, D.C., et al. 2004. Polymorphism in the Plasmodium falciparum erythrocyte-binding ligand JESEBL/EBA-181 alters its receptor specificity. Proc. Natl. Acad. Sci. U. S. A. 101:2518-2523.

96. Maier, A.G., Baum, J., Smith, B., Conway, D.J., and Cowman, A.F. 2009. Polymorphisms in erythrocyte binding antigens 140 and 181 affect function and binding but not receptor specificity in Plasmodium falciparum. Infect. Immun. 77:1689-1699.

97. Gaur, D., et al. 2006. Upregulation of expression of the reticulocyte homology gene 4 in the Plasmodium falciparum clone Dd2 is associated with a switch in the erythrocyte invasion pathway. Mol. Biochem. Parasitol. 145:205-215.

98. Stubbs, J., et al. 2005. Molecular mechanism for switching of $P$. falciparum invasion pathways into human erythrocytes. Science. 309:1384-1387.

99. Hayton, K., et al. 2008. Erythrocyte binding protein PfRH5 polymorphisms determine species-specific pathways of Plasmodium falciparum invasion. Cell Host Microbe. 4:40-51.

100.Suwanarusk, R., et al. 2004. The deformability of red blood cells parasitized by Plasmodium falciparum and P. vivax. J. Infect. Dis. 189:190-194.

101.Galinski, M.R., Medina, C.C., Ingravallo, P., and Barnwell, J.W. 1992. A reticulocyte-binding protein complex of Plasmodium vivax merozoites. Cell. 69:1213-1226.

102.Miller, L.H., Mason, S.J., Clyde, D.F., and McGinniss, M.H. 1976. The resistance factor to Plasmodium vivax in blacks. The Duffy-blood-group genotype, FyFy. N. Engl. J. Med. 295:302-304.

103.Zimmerman, P.A., et al. 1999. Emergence of FY*A(null) in a Plasmodium vivax-endemic region of Papua New Guinea. Proc. Natl. Acad. Sci. U. S. A. 96:13973-13977.

104.Kasehagen, L.J., et al. 2007. Reduced Plasmodium vivax erythrocyte infection in PNG Duffy-negative heterozygotes. PLoS ONE. 2:e336.

105.Galinski, M.R., and Corredor, V. 2004. Variant antigen expression in malaria infections: posttranscriptional gene silencing, virulence and severe pathology. Mol. Biochem. Parasitol. 134:17-25.

106.Carter, R., and Mendis, K.N. 2002. Evolutionary and historical aspects of the burden of malaria. Clin. Microbiol. Rev. 15:564-594.

107. Trape, J.F., et al. 1998. Impact of chloroquine resistance on malaria mortality. C. R. Acad. Sci. III. 321:689-697.

108. Rice, L.B. 2008. Federal funding for the study of antimicrobial resistance in nosocomial pathogens: no ESKAPE. J. Infect. Dis. 197:1079-1081.

109. Hayton, K., Fairhurst, R.M., Naude, B., Su, X.Z., and Wellems, T.E. 2005. Drug-resistant falciparum malaria: mechanisms, consequences, and challenges. In Frontiers in antibiotic resistance: a tribute to Stuart B. Levy. ASM Press. Washington, DC, USA. 401-413.

110.Nomura, T., et al. 2001. Evidence for different mechanisms of chloroquine resistance in 2 Plasmodium species that cause human malaria. J. Infect. Dis. 183:1653-1661.

111.Laufer, M.K., et al. 2006. Return of chloroquine antimalarial efficacy in Malawi. N. Engl. J. Med. 355:1959-1966.

112.Wang, X., et al. 2005. Decreased prevalence of the Plasmodium falciparum chloroquine resistance transporter 76T marker associated with cessation of chloroquine use against $P$. falciparum malaria in Hainan, People's Republic of China. Am. J. Trop. Med. Hyg. 72:410-414

113.Li, Y., Huang, H., and Wu, Y.-L. 2006. Quinghaosu (Artemsinin) - a fantastic antimalarial drug from a traditional Chinese medicine. In Medicinal chemistry of bioactive products. X.-T. Liang and W.-S. Fang, editors. John Wiley and Sons. 183-256.

114. Noedl, H., et al. 2008. Evidence of artemisinin-resistant malaria in western Cambodia. N. Engl. J. Med. 359:2619-2620.

115.White, N.J. 2008. Qinghaosu (artemisinin): the price of success. Science. 320:330-334.

116. Bejon, P., et al. 2008. Efficacy of RTS, S/AS01E vaccine against malaria in children 5 to 17 months of age. N. Engl. J. Med. 359:2521-2532.

117. Sachs, J., and Malaney, P. 2002. The economic and social burden of malaria. Nature. 415:680-685.

118.Sinden, R.E. 1998. Gametocytes and sexual development. In Malaria: parasite biology, pathogenesis, and protection. I.W. Sherman, editor. ASM Press. Washington, DC, USA. 25-47.

119.Templeton, T.J. 2007. Whole-genome natural histories of apicomplexan surface proteins. Trends Parasitol. 23:205-212.

120.Escalante, A.A., and Ayala, F.J. 1995. Evolutionary origin of Plasmodium and other Apicomplexa based on rRNA genes. Proc. Natl. Acad. Sci. U. S. A. 92:5793-5797.

121. Greenwood, B., Marsh, K., and Snow, R. 1991. Why do some African children develop severe malaria? Parasitol. Today. 7:277-281.

122.Mehlotra, R.K., et al. 2008. Discordant patterns of genetic variation at two chloroquine resistance loci in worldwide populations of the malaria parasite Plasmodium falciparum. Antimicrob. Agents Chemother. 52:2212-2222.

123.Zakeri, S., et al. 2008. Association of $p f c r t$ but not $p f m d r 1$ alleles with chloroquine resistance in Iranian isolates of Plasmodium falciparum. Am. J. Trop. Med. Hyg. 78:633-640.

124.Luzzatto, L., Nwachuku-Jarrett, E.S., and Reddy, S. 1970. Increased sickling of parasitised erythrocytes as mechanism of resistance against malaria in the sickle-cell trait. Lancet. 1:319-321.

125.Roth, E.F., Jr., et al. 1978. Sickling rates of human AS red cells infected in vitro with Plasmodium falciparum malaria. Science. 202:650-652.

126.Friedman, M.J. 1978. Erythrocytic mechanism of sickle cell resistance to malaria. Proc. Natl. Acad. Sci. U. S. A. 75:1994-1997.

127. Cockburn, I.A., et al. 2004. A human complement receptor 1 polymorphism that reduces Plasmodium falciparum rosetting confers protection against severe malaria. Proc. Natl. Acad. Sci. U. S. A. 101:272-277.

128.Fowkes, F.J., et al. 2008. Increased microerythrocyte count in homozygous alpha(+)-thalassaemia contributes to protection against severe malarial anaemia. PLoS Med. 5:e56. 